\title{
Zur Kenntniss der aus Berberin entstehenden Pyridinearbonsäuren
}

\author{
von
}

Richard Mayer.

Aus dem I. chemischen Universitätslaboratorium in Wien.

(Vorgelegt in der Sitzung am 17. März 18921)

Ausser den sechs, der Theorie nach möglichen Pyridindicarbonsäuren sind noch deren drei dargestellt und als selbstständige Verbindungen beschrieben worden.

Eine derselben wurde von $\mathrm{H}$. Fürth ${ }^{1}$ aus Berberonsäure erhalten, jedoch in so geringen Quantitäten, dass die Zurückführung auf eine der sechs bekannten Pyridindicarbonsäuren nicht gelang.

Ich habe diese Säure, die in der Literatur vielfach Beronsäure genannt wird, untersucht und ihre Identificirung durchgeführt.

Das Ausgangsmaterial für die Gewinnung derselben, die Berberonsäure, wurde zuerst von $\mathrm{Weide} 1^{2}$ dargestellt und als Pyridintricarbonsäure erkannt. Sie bildet tricline Prismen, die lufttrocken zwei, mitunter auch ein Molecül Krystallwasser enthalten, verwittert sehr rasch an der Luft und wird in wässeriger Lösung von schwefelsaurem Eisen intensiv roth gefärbt. Das, mit Ätzkalk destillirte Kalksalz liefert Pyridin. Ihre Tribasicität stellte Fürth ${ }^{3}$ durch Untersuchung der möglichen Salzreihen fest; zugleich trachtete er durch pyrogenetische Zersetzung sowohl der freien Säure, wie auch ihrer Salze zu einer Pyridin-

\footnotetext{
1 Monatsh. f. Chem. 2, S. 426.

2 Ber. d. k. Akad. 78, 2. Abth. Juniheft.

3 Monatsh. f. Chem. 2. S. 416 u. ff.
} 
dicarbonsäure zu gelangen, ohne jedoch ein anderes Product als Pyridinmonocarbonsäuren erhalten zu können. Unterdessen hatten Hoogewerf und van Dorp ${ }^{1}$ die Beobachtung gemacht, dass aus mehrbasischen Carbonsäuren desPyridins beim Kochen mit Eisessig solche von geringerer Basicität oft erhältlich sind. Dieses Verfahren erwies sich als erfolgreich bei Berberonsäure und lieferte eine Pyridindicarbonsäure, die Fürth folgendermassen beschreibt: Wasserfreie, farblose Krystalle, in kaltem Wasser wenig, in heissem leicht löslich, die von Alkohol, auch beim Erhitzen nur in sehr geringer Menge aufgenommen werden. Gibt mit salpetersaurem Silber einen weissen, mit essigsaurem Kupfer in heisser Lösung einen blaugrünen Niederschlag, welch letzterer sich beim Erkalten auflöst. In wässerigerLösung mit schwefelsaurem Eisen keine Färbung. Schmelzpunkt $263^{\circ}$ Die feuchte Substanz färbt sich an der Luft roth. Er erkannte sie als Pyridindicarbonsäure und stellte ihr saures Kaliumsalz dar, das mit der Säure die Eigenschaft des Rothwerdens an der Luft gemeinsam hat. Er schloss aus alledem, dass er es hier mit einer, bisher unbekannten Säure zu thun habe.

Behufs Gewinnung derselben musste ich erst Berberonsäure darstellen; da ich hiebei einige, von den bisherigen Angaben abweichende Beobachtungen machte, so will ich in Kürze den von mir eingehaltenen Weg beschreiben.

Käufliches Berberin wurde in vier Partien, jede zu circa $50 \mathrm{~g}$ in einem geräumigen Kolben der Oxydation unterworfen. Nachđem die stürmische Einwirkung der Salpetersäure nachgelassen hatte, wurde solange unter Erneuern der verdampften Säure gekocht, bis die Flüssigkeit nur mehr weingelb erschien. Dann entfernte ich durch Abdestilliren den grössten Theil der Salpetersäure und erhielt aus der verbleibenden syrupartigen Flüssigkeit gelblich gefärbte Krystalle $(A)$, deren Mutterlauge weiter oxydirt wurde.

Meine so gewonnene Ausbeute betrug circa $14 \%$ der angewendeten Menge Berberin.

Die Theile, aus denen keine Krystalle mehr zu erhalten waren, vereinte ich und versetzte sie mitBarytwasser. Der zuerst

1 Berl. Ber. XIV, S. 974. 
herausfallende reichliche Niederschlag gab, mit Schwefelsäure zersetzt, noch Berberonsäure. Aus der Mutterlauge, die von demselben abfiltrirt worden war, erhielt ich nach Behandlung mit Schwefelsäure eine weitere Menge einer krystallinischen Sub$\operatorname{stanz}(B)$; die nicht mehr die rothe Reaction mit schwefelsaurem Eisen zeigte.

Die so hergestelite Berberonsäure hatte den Schmelzpunkt $234-235^{\circ}$, gab die rothe Eisenreaction und das in der Kälte lösliche, in der Hitze unlösliche.Kupfersalz. Von Krystallwasser wurden sowohl eines als auch zwei Molecüle gefunden. Ihre Eigenschaften stimmen demnach mit der Berberonsäure, die Fürth's Ausgangsproduct bildete bis auf den Schmelzpunkt, den er zu $243^{\circ}$ angibt, überein. Da durch blosses Umkrystallisiren der der Säure zäh anhängende gelbe Körper sehr schwer wegzubringen ist, löste ich dieselbe in concentrirter Salzsäure und fällte sie daraus durch Einleiten von gasförmiger Salzsäure, wobei ich eine rein weisse Substanz erhielt, die nach dem Umkrystallisiren aus Wasser wieder den Schmelzpunkt $235^{\circ}$ zeigte; denselben fand ich auch bei Berberonsäure, die ich durch Zersetzung ihrer Salze gewonnen hatte.

\section{Darstellung der Pyridindicarbonsäure aus Berberonsäure.}

Zur Herstellung der Pyridindicarbonsäure aus Berberonsäure, welch erstere ich mit dem ihr oft beigelegten Namen Beronsäure der Kürze wegen im Folgenden bezeichne, wurde ein Gemenge von $10 \mathrm{~g}$ Berberonsäure, Essigsäure und Essigsäure-Anhydrid (2:1) durch sechs Stunden auf Siedetemperatur erhitzt, wobei Kohlensäure-Abspaltung durch vorgelegtes Barytwasser nachzuweisen war.

Da nach beendetem Erhitzen noch unveränderte Berberonsäure an der Reaction mit schwefelsaurem Eisen zu erkennen war, brachte ich das ganze Gemisch in. Röhren, die zugeschmolzen und durch sechs Stunden auf $160^{\circ}$ erhitzt wurden. Das Reactionsproduct löste ich nach dem Vertreiben der Essigsäure in viel heissem Wasser und versetzte es in einer geräumigen Schale mit reinem Bleicarbonat. Der Niederschlag der aus der braunen Flüssigkeit herausfiel, wurde abfiltrirt und so 
lange mit heissem Wasser gewaschen, bis das Waschwasser mit Schwefelwasserstoff nur mehr eine leichte Braunfärbung erkennen liess. Das Bleisalz am Filter war gelblich, das Filtrat braun. Dieses, mit Schwefelwasserstoff zersetzt, lieferte eine sehr geringe Menge einer bräunlichen Substanz, deren Lösung mit Kupferacetat einen in der Hitze unlöslichen, in der Kälte löslichen, grünen Niederschlag gab. Es war demnach bei dieser Reaction keine irgendwie erheblichere Menge von Monopyridincarbonsäure entstanden. Das unlösliche Bleisalz, das am Filter geblieben war, zersetzte ich nach dem Suspendiren in Wasser durch Einleiten von Schwefelwasserstoff. Dem Schwefelblei konnten durch mehrmaliges Auskochen noch zurückgehaltene Substanzmengen entzogen werden. Die, nach dem Einengen der so erhaltenen Lösungen, herausfallenden Krystalle waren gelb, liessen sich jedoch mittelst Blutkohle sehr leicht reinigen, und bildeten weisse, glänzende Nadeln vom Schmelzpunkt $249^{\circ}$. Nach dem Gange der Darstellung müssten nun diese Krystalle der Beronsäure entsprechen. In der That bemerkte ich auch, dass deren Mutterlauge beim Stehen an der Luft zunächst am Rande, später durch die ganze Flüssigkeit roth wurde. Ebenso war es auch mit feuchter Substanz; jedoch die reinste Partie mit dem constanten Schmelzpunkt von $249^{\circ}$ zeigte diese Erscheinung nur in sehr geringem Masse. Die Elementaranalyse ergab folgende Werthe:

$0.2007 g$ Substanz lieferten $0.3674 g$ Kohlensäure und $0.0474 g$ Wasser.

In 100 Theilen:

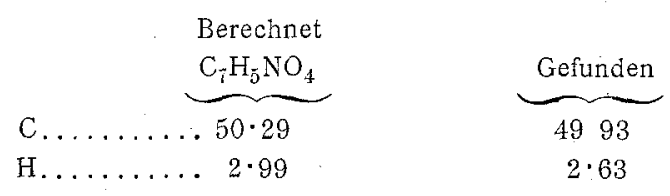

Sie enthält kein Krystallwasser.

Die Eigenschaften, die Fürth für Beronsäure angibt: wasserfreie, farblose Krystalle, in kaltem Wasser wenig, in heissem leicht, in Alkohol schwer löslich, mit schwefelsaurem Eisen keine Färbung, Rothfärbung in feuchtem Zustande an der 
Luft, sowie die Analyse, finden sich an der von mir dargestellten Säure alle wieder, hingegen differiren die Schmelzpunkte (bei Fürth $263^{\circ}$, bei mir $249^{\circ}$ ) beträchtlich.

Nun werde ich im Folgenden den Nachweis führen, dass die Beronsäure identisch ist mit Cinchomeronsäure und gerade für diese Säure existiren dreierlei verschiedene Angaben betreffs des Schmelzpunktes, von welchen sich zwei mit der meinigen und eine andere mit der Fürth's decken. Nämlich: Weidel und Schmidt, ${ }^{1}$ wie auch Hoogewerf und van Dorp $249-251^{\circ}$, Skraup ${ }^{3} 258-259^{\circ}$, Öchsner de Coninck $266-268^{\circ}$ (bei $250^{\circ}$ beginnend). Bei einer so grossen Divergenz der Angaben kann wohl diesem Unterschiede zwischen der von mir dargestellten Säure und der Beronsäure keine grosse Bedeutung beigelegt werden, umsoweniger, als diese Schmelzpunkte eigentlich Zersetzungspunkte sind, dieje nachdem rascher oder langsamer erhitzt wird, sich nach oben oder nach unten verschieben.

Die soeben beschriebenen Eigenschaften dieser Säure passen vollkommen auf Cinchomeronsäure. Nun gibt die letztere mit Salzsäure eine eminent krystallisirende Verbindung, die zuerst von Skraup dargestellt und von v. Lang gemessen wurde.

Sie schien mir besonders geeignet, um damit den Nachweis der Identität von Beronsäure mit Cinchomeronsäure zu führen.

Ich habe zu diesem Behufe den reinsten Theil der Säure: (von dem eine Partie zur Analyse gedient hatte) in Salzsäure aufgelöst und diese Lösung langsam über Schwefelsäure abdunsten lassen. Es schieden sich fächerförmig angeordnete: Täfelchen aus, die trotz aller Vorsicht nicht in einen, für die Messung brauchbaren Zustand gebracht werden konnten. Erst nachdem ich diese Krystalle neuerdings in etwas verdünnterer Salzsäure gelöst hatte, erhielt ich nach allmälichem Abdunsten wohlausgebildete Krystalle, die Herr Dr. R. Köchlin, dem ich für seine liebenswürdige Mühewaltung hier meinen Dank.

\footnotetext{
1 Berl. Ber. XII, S. 1148.

2 Ann. Chem. 204, S. 84.

3 Berl. Ber. XV, S. 1507.

4 Compt. rend. 91, S. 296, und Ber1. Ber. XIII, S. 1996.
} 
ausspreche, so freundlich war zu messen. Er theilt mir hierüber Folgendes mit:

»Die mir zur Messung behufs Constatirung der Identität mit den von Lang gemessenen Krystallen der Verbindung $\mathrm{C}_{7} \mathrm{H}_{5} \mathrm{NO}_{4} \cdot \mathrm{HCl}$ übergebenen Krystalle sind monosymmetrisch und zeigen folgende Combination:

$$
\begin{aligned}
& \begin{array}{lllll}
(100) & (001) & (110) & (120) & (590)
\end{array} \\
& \text { Lang fand - (001) (110) - (590) }
\end{aligned}
$$

überdies konnte ich eine nicht messbare Pyramide in der Zone $\{110.001\}$ beobachten. Die Krystalle sind ausgezeichnet spaltbar nach (100), unvollkommen nach (110). Die Messungen von neun Krystallen ergaben im Mittel:

\begin{tabular}{|c|c|c|c|}
\hline & \multirow{2}{*}{$\begin{array}{c}\text { Köchlin } \\
\text { gemessen }\end{array}$} & \multicolumn{2}{|c|}{ von Lang } \\
\cline { 2 - 4 } & gemessen & berechnet \\
\hline \hline$(110):(110)$ & $86^{\circ} 13^{\prime}$ & $86^{\circ} 44^{\prime}$ & - \\
$(110):(120)$ & $18^{\circ} 42^{\prime}$ & - & $18^{\circ} 431^{\prime} / 3$ \\
$(110):(590)$ & $16^{\circ} 27^{\prime}$ & $16^{\circ} 8^{\prime}$ & $16^{\circ} 10^{\prime}$ \\
$(110):(001)$ & $82^{\circ} 30^{\prime}$ & $82^{\circ} 56^{\prime}$ & - \\
\hline
\end{tabular}

- Die von Lang gerechneten Elemente sind: $a: b: c=$ $0.9584: 1:$ ? $a c=99^{\circ} 45^{\prime}$.

Nachdem die Krystalle fächerförmige Aggregate sind und infolge dessen sehr schwankende und unsichere Werthe ergeben, kann die Übereinstimmung der Winkel von Lang's mit meinen als genügend und die Identität als festgestellt betrachtet werden. «

Von der Substanz, deren einer Theil gemessen worden war, wurde ein anderer zu einer Verbrennung und ein dritter zur Chlorbestimmung verwendet.

I. $0 \cdot 2130 g$ Substanz gaben $0 \cdot 3216 g$ Koh lensäure und $0.0605 g$ Wasser II. $0 \cdot 2130 \mathrm{~g}$ Substanz gaben $0 \cdot 1474 \mathrm{~g}$ Chlorsilber. 
In 100 Theilen:

\begin{tabular}{|c|c|c|c|c|}
\hline & $\begin{array}{r}\mathrm{Ber} \\
\mathrm{C}_{7} \mathrm{H}_{5} \\
\end{array}$ & $\begin{array}{l}\text { et } \\
\mathrm{HCl}\end{array}$ & \multicolumn{2}{|c|}{ Gefunden } \\
\hline & I & II & I & II \\
\hline C. & $41 \cdot 27$ & - & $41 \cdot 18$ & - \\
\hline $\mathrm{H}$ & $2 \cdot 94$ & - & $3 \cdot 15$ & - \\
\hline $\mathrm{Cl}$ & - & $17 \cdot 44$ & - & $17 \cdot 12$ \\
\hline
\end{tabular}

Es ist demnach die Beronsäure identisch mit Cinchomeronsäure.

Diese Säure ist als Zersetzungsproduct dreier verschiedener Pyridintricarbonsäuren möglich, nämlich derjenigen, welche in Stellung $\beta$ und $\gamma j$ je ein Carboxyl haben. Die dem dritten Carboxyl in der Berberonsäure anzuweisenden Stellungen sind durch die Thatsache, dass diese Säure mit schwefelsaurem Eisen die bekannte rothe Reaction gibt, auf die zwei, dem Stickstoff benachbarten beschränkt. Da nun aber feststeht, dass die Pyridintricarbonsäure $(\alpha \beta \gamma)$ mit Berberonsäure nicht identisch ist, bleibt als einzige zulässige Annahme für die letztere, dass ihre Carboxyle in $\alpha^{\prime} \beta \gamma$ angeordnet sind.

Ich habe mich bemüht, das Resultat dieserSchlussfolgerung experimentell zu bekräftigen, indem ich versuchte eine Spaltung der Berberonsäure in einem, diesem Zwecke dienlichen Sinne durchzuführen. Da mir dieselbe nur in geringen Quantitäten zur Verfügung stand, machte ich zunächst mit Cinchomeronsäure einen orientirenden Versuch.

\section{Zerfall der Cinchomeronsäure beim Erhitzen mit Chinolin.}

Wie bekannt, liefert diese Säure, für sich erhitzt, ein Gemenge von Isonicotinsäure und Nicotinsäure. ${ }^{1}$ Ihr saures Natriumsalz zerfällt nach $\mathrm{Skraup}{ }^{2}$ bei höherer Temperatur unter Bildung von Isonicotinsäure ( $\gamma$ Pyridincarbonsäure).

Diese Zersetzungen liefern beide die Isonicotinsäure in ziemlich geringen Mengen, und zwar erhält man beim Erhitzen der Cinchomeronsäure für sich kaum mehr, als $20 \%$ an

${ }^{1}$ H o ogewerf und van Dorp, Ann. Chem. 207, S. 221.

2 Akad. Ber. 
Isonicotinsäure, während die Hauptmenge Nicotinsäure ist. Nicht viel günstiger ist die Ausbeute beim Erhitzen des sauren Natriumsalzes, denn auch hier werden nur circa $25 \%$ an Isonicotinsäure gebildet, indem hauptsächlich Nicotinsäure entsteht.

Das Erhitzen der Cinchomeronsäure mit Essigsäure und Essigsäure - Anhydrid, wobei ebenfalls eine totale Spaltung in Pyridinmonocarbonsäuren eintritt, liefert vorwiegend Nicotinsäure.

Günstigere Resultate habe ich erzielt durch Erwärmen der Cinchomeronsäure mit Chinolin.

$3.5 g$ der Säure mit $18 g$ vollkommen trockenen und durch Destillation gereinigten Chinolins wurden am Rückflusskühler erhitzt. Zunächst erfolgte Lösung der Säure und bei circa $200^{\circ}$ war ein leichtes Aufschäumen und Trübung des vorgelegten Barytwassers zu bemerken. Nach beendeter Reaction wurde das überschüssige Chinolin durch Wasserdampf abgetrieben und die zurückbleibende dunkelbraune Masse nach dem Ansäuern mit Salzsäure mit Thierkohle entfärbt. Aus der klaren Lösung erhielt ich Krystalle vom Schmelzpunkt $305^{\circ}$ und in bei weitem geringerer Menge solche von niedrigerem Schmelzpunkte.

Die ersteren wurden analysirt und zwar gaben: $0 \cdot 2954 g$ Substanz, $0 \cdot 6321 g$ Kohlensäure und $0 \cdot 1076 g$ Wasser.

In 100 Theilen:

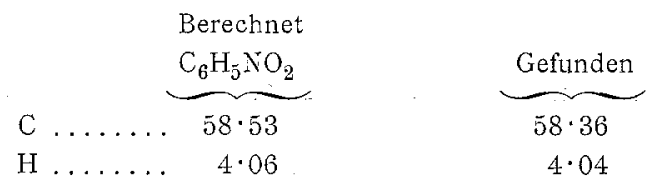

Kein Krystallwasser.

Das Reactionsproduct war demnach Isonicotinsäure und erreichte circa $34 \%$ der in Arbeit genommenen Menge Cinchomeronsäure.

\section{Zersetzung des Dinatriumsalzes der Berberonsäure.}

Der Verlauf der Zersetzung von Cinchomeronsäure in Chinolin liess hoffen, dass Berberonsäure, analog behandelt, 
Producte liefern würde, die zum Nachweis der Stellung ihres $\alpha$ Carboxyls tauglich wären. Andererseits stand $z u$ befürchten, dass die Zersetzung zu weit gehen könnte, selbst bei sorgfältiger Regulirung der Temperatur, und zum Constitutionsnachweis unbrauchbare Monocarbonsäuren entstehen würden.

Deshalb zog ich vor zwei Carboxyle der Berberonsäure mit Natrium abzusättigen, so dass nur das dritte, freie Carboxyl in Reaction treten konnte.

Es wurden deshalb $9 \mathrm{~g}$ Berberonsäure auf das Dinatriumsalz verarbeitet, dieses bei $180^{\circ}$ getrocknet, mit der fünffachen Menge reinen, trockenen Chinolins versetzt und auf Siedetemperatur des letzteren durch sechs Stunden erhalten. Dabei war die Kohlensäureentwickelung sehr gering und die Aufarbeitung des Reactionsproductes ergab eine Säure, die ein Molecül Krystallwasser enthielt und bei der Analyse die folgenden Werte lieferte:

$0.2378 g$ Substanz gaben $0.3954 g$ Kohlensäure und $0 \cdot 0499 g$ Wasser.

In 100 Theilen:

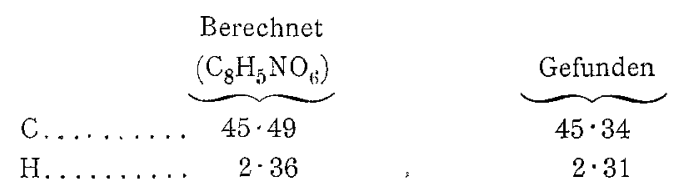

Der Schmelzpunkt war $234^{\circ}$. Es war also die Berberonsäure unverändert geblieben.

Da dies offenbar auf die zu niedrige Temperatur zurückzuführen war, wiederholte ich den Versuch mit Zusatz von Diphenylamin, um durch Steigerung der Siedetemperatur eine energischere Reaction zu erreichen. Es wurde hiedurch eine Erhöhung des Siedepunktes auf $260^{\circ}$ bewirkt.

Als der vorgelegte Kaliapparat nur mehr sehr unbedeutende Gewichtszunahme erkennen liess, stellte ich das Erhitzen ein. Beim Auseinandernehmen des Apparates war in dem Kolben, der das Reactionsproduct enthielt, starker Pyridingeruch wahrzunehmen. Die Schwefelsäure, durch welche die Kohlensäure streichen musste, bevor sie zur Kalilauge gelangte, gab 
mit Alkali im Überschuss versetzt und erhitzt, diesen Geruch in noch bedeutenderem Maasse.

Chinolin und Diphenylamin, durch Extraction mittelst Äther entfernt, liessen eine dunkelbraune Substanz zurück, die bis auf einen geringen Rest einer schmierigen Masse in heissem Wasser löslich war. Die Lösung gab mit Bleiacetat eine gelbliche Fällung, die nach dem Zersetzen mit Schwefelwasserstoff eine weisse krystallinische Substanz lieferte. Nach dem Umkrystallisiren aus Wasser wurden Krystalle von dem Schmelzpunkt $234^{\circ}$ erhalten. Sie hatten ein Molekül Krystallwasser und gaben in wässeriger Lösung die rothe Reaction mit schwefelsaurem Eisen.

Bei der Elementaranalyse fand ich, dass $0.2237 \mathrm{~g}$ der Substanz $0.3824 g$ Kohlensäure und $0.566 g$. Wasser lieferten.

In 100 Theilen:

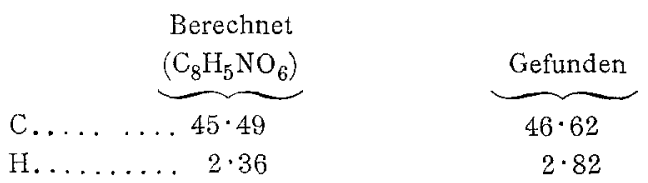

Die Analyse zeigt, dass hier ein Gemenge von unveränderter Berberonsäure mit Pyridindicarbonsäure oder mit Pyridinmonocarbonsäure vorliegt.

Aus diesem Gemisch versuchte ich auf verschiedene Weise die Componenten rein $\mathrm{zu}$ erhalten. Unter anderem habe ich mich bemüht, Cinchonmeronsäure daraus abzuscheiden mit Hilfe ihrer Salzsäureverbindung, ohne jedoch dabei etwas zu erreichen.

Weiters habe ich das Gemenge in Salzsäure gelöst, mit Platinchlorid versetzt und längere Zeit stehen lassen. Hiebei hat sich ein geringes Quantum gelblicher Krystalle abgeschieden, die ihrem Aussehen nach als Platindoppelverbindung von Pyridinmonocarbonsäure anzusehen sind.

Jedenfalls gelang es durch Behandlung des Dinatriumsalzes mit Chinolin nicht, die Spaltung in einem anderen Sinne herbeizuführen, da, wie die Versuche gezeigt haben, bei der angewendeten Temperatur die Berberonsäure eine tiefergehende 
Zersetzung erleidet, indem ein Theil derselben total zerfällt unter Bildung von Pyridin und Kohlensäure.

\section{Untersuchung von $B$.}

Bei der Oxydation von Berberin bildet sich nach Angabe Weidel's ausser Berberonsäure noch eine sehr geringe Menge eines Nitroproductes. Dieser Körper, der offenbar seiner gelben Farbe wegen für einen Nitrokörper gehalten wurde, ist nie genau untersucht worden. Da nun die gelb gefärbte Partie $B$ immerhin in solcher Menge erhalten wurde $(5 \mathrm{~g})$, dass sich eine nähere Untersuchung lohnte, beschäftigte ich mich eingehender mit derselben, umsomehr, als darin der Nitrokörper vermuthet werden musste.

Sie erwies sich als löslich in heissem Wasser, wurde von Alkohol wenig, Äther gar nicht aufgenommen. Viermalige Extraction mit heissem.Alkohol gab eine gelb gefärbte Lösung und einen schwach gelblichen Rückstand. Dieser, aus Wasser umkrystallisirt, lieferte gelbe Nadeln, von welchen sich auch aus dem alkoholischen Extract eine kleine Menge isoliren liess. Aus diesen Nadeln erhielt ich nach dem Reinigen mit Blutkohle eine weisse krystallinische Substanz von dem Schmelzpunkt $249^{\circ}$, die bei $100^{\circ}$ keinen Gewichtsverlust zeigte:

$0 \cdot 2002 g$ Substanz gaben bei der Verbrennung $0.3592 g$ Kohlensäure und $0 \cdot 0545 \mathrm{~g}$ Wasser.

In 100 Theilen:

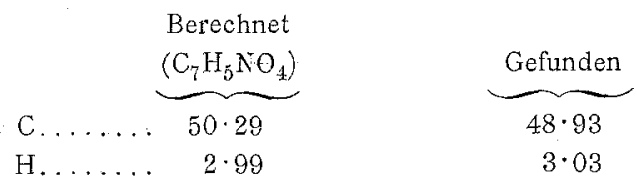

Die wässerige Lösung reagirte nicht mit schwefelsaurem Eisen. Da die erhaltenen Resultate nicht genügten, um die vermuthete Identität mit Cinchomeronsäure nachzuweisen, verwerthete ich die früher gemachten Erfahrungen über Zersetzung dieser Säure in Chinolin, um den erstrebten Nachweis zu führen. $21 / 2 g$ der Substanz kamen in Verwendung und lieferten ein Reactionsproduct, das nach dem Umkrystallisiren aus Wasser, 
soweit der Schmelzpunkt ein Urtheil erlaubte, aus Isonicotinsäure bestand, die mit etwas Nicotinsäure vermengt war. Ich benützte die, von Weidel und Herzig ${ }^{1}$ angegebene Thatsache, dass die erstere in Alkohol schwer, die andere leicht löslich ist, zur weiteren Trennung und erhielt Krystalle von dem Schmelzpunkt 305-306 ${ }^{\circ}$.

$0.2518 \mathrm{~g}$ dieser Substanz gaben bei der Elementaranalyse $0.5389 \mathrm{~g}$ Kohlensäure und $0.0995 \mathrm{~g}$ Wasser.

In 100 Theilen:

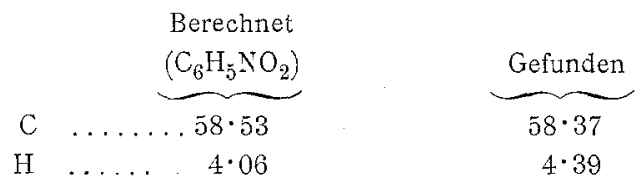

Sie enthält kein Krystallwasser.

Die so erhaltene Isonicotinsäure gestattet den Schluss, dass der Theil $B$ unreine Cinchomeronsäure war.

$\mathrm{Da}$ trotz aller Sorgfalt kein Nitrokörper zu finden war, kann wohl angenommen werden, dass bei der Oxydation von Berberin mit Salpetersäure kein solcher entstanden war.

Das Auftreten der Cinchomeronsäure als Oxydationsproduct des Berberins kann man entweder durch die Annahme erklären, dass sie direct aus diesem entsteht, oder auch, dass die zunächst gebildete Berberonsäure bei anhaltendem Kochen mit Salpetersäure, ganz so, wie beim Erhitzen mit Essigsäure, in Cinchomeronsäure und Kohlensäure zerfällt.

W. H. Perkin ${ }^{2}$ jun. hat aus den Resultaten, die er bei Oxydation des Berberins erhielt, den Schluss gezogen, dass dieses Alkaloïd in Bezug auf seine Constitution mit Papaverin ${ }^{3}$ und Narcotin ${ }^{4}$ verwandt, und schliesslich als ein Derivat des Isochinolins zu betrachten ist.

\footnotetext{
1 Monatsh. f. Chem. 1, S. 41.

2 Berl. Ber. XXII, Ref. 294.

3 Goldschmie d, Monatsh. f. Chem. 9, S. 350.

4 Ros er, Ann. Chem. 254, S. 357.
} 
Diese Annahme findet in der Bildung der Berberonsäure, welche als Spaltungsproduct Cinchomeronsäure liefert, und in dem Umstande, dass bei der Oxydation des Berberins mit Salpetersäure möglicherweise auch direct diese Pyridindicarbonsäure entsteht, insofern eine Bestätigung, als man weiss, dass Isochinolin, beziehungsweise dessen Derivate bei der Oxydation auch Cinchomeronsäure liefern.

Ich erfülle schliesslich eine Pflicht aufrichtiger Dankbarkeit, indem ich Herrn Prof. Weidel für seine vielfache, werthvolle Unterstützung, und die guten Rathschläge, durch die er mich in der Arbeit förderte, an dieser Stelle meinen Dank ausspreche. 